\title{
On the abnormal structure of finite groups
}

\author{
Adolfo Ballester-Bolinches, John Cossey \\ and Ramón Esteban-Romero
}

\begin{abstract}
We study finite groups in which every maximal subgroup is supersoluble or normal. Our results answer some questions arising from papers of Asaad and Rose.
\end{abstract}

\section{Introduction and statement of results}

In this paper we will consider only finite groups.

A classical result of Schmidt [17] shows that if every maximal subgroup of a group is nilpotent, then the group is soluble. Rose [16] considered the effects of replacing "maximal" by "non-normal maximal" in Schmidt's result, and proved:

Theorem 1. If every non-normal maximal subgroup of a group $G$ is nilpotent, then $G$ has a normal Sylow subgroup $P$ such that $G / P$ is nilpotent.

It is clear that the hypothesis in the above theorem holds in every epimorphic image of $G$. Hence, using induction on the order of $G$, the solubility of the group is a consequence of the following result proved by Baer in [4]:

Theorem 2. Let $G$ be a primitive group such that every core-free maximal subgroup is nilpotent. Then $G$ is soluble.

Among the published extensions of Schmidt's result, one due to Huppert is of particular interest. He proved:

Theorem 3 ([11, Satz 22]). If every maximal subgroup of a group $G$ is supersoluble, then $G$ is soluble.

Rose [16] observed that imposing supersolubility only on non-normal maximal subgroups is not sufficient to guarantee solubility. He shows that in $\mathrm{PGL}_{2}(7)$, every maximal subgroup except $\mathrm{PSL}_{2}(7)$ is supersoluble. Hence before the classification 
of simple groups, there was no hope of describing these groups. In fact, this classification is used by $\mathrm{Li}$ and Shi [14] to prove a result from which the following theorem is an immediate consequence:

Theorem 4. If every non-normal maximal subgroup of a group $G$ is supersoluble, then the composition factors of $G$ are isomorphic to $\mathrm{PSL}_{2}(p)$ or $C_{q}$, where $p$ and $q$ are primes and $p^{2}-1 \equiv 0(\bmod 16)$.

However, a structural description of these groups remains open. In this context, Asaad [2] asked for a supersoluble version of Theorem 2:

Question 5. What can be said about the structure of a primitive group in which all core-free maximal subgroups are supersoluble?

The main aim of this paper is to present answers to these questions. We prove:

Theorem A. Let $G$ be a group. Then every non-normal maximal subgroup of $G$ is supersoluble if and only if $G$ satisfies one the following conditions:

1. If $G$ is insoluble, then the following conditions hold:

(a) $G / \mathrm{F}(G) \cong \mathrm{PGL}_{2}(p)$, where $p$ is a prime such that $p^{2}-1 \equiv 0(\bmod 16)$,

(b) the soluble residual and the nilpotent residual of $G$ coincide and are isomorphic to $\mathrm{PSL}_{2}(p)$ or $\mathrm{SL}_{2}(p)$.

2. Assume that $G$ is soluble, but not supersoluble. Let $A$ be the supersoluble residual of $G$ and let $Z$ be the supersoluble hypercentre of $G$.

(a) $A$ is a p-group for a prime $p, A / A^{\prime}$ is a complemented non-cyclic chief factor of $G$, and all chief factors of $G$ containing $A$ or contained in $A^{\prime}$ are cyclic. Moreover, $Z$ contains no non-central complemented chief factors of $G$ and $A$ has nilpotency class at most two.

(b) Either

i. $G / \mathrm{C}_{G}\left(A / A^{\prime}\right)$ is nilpotent, or

ii. $G / \mathrm{C}_{G}\left(A / A^{\prime}\right)$ is isomorphic to a nontrivial semidirect product of the form $[B](T \times P)$, where $T$ is an abelian group of exponent dividing $p-1, P$ is a p-group, $B$ is a cyclic group of order $q$ for a prime $q \neq p$, and the nilpotent residual of $G / A$ is a q-group.

3. $G$ is supersoluble.

Theorem B. Let $G$ be a primitive group. Then every core-free maximal subgroup of $G$ is supersoluble if and only if $G$ is either $G \cong \operatorname{PGL}_{2}(p)$ with $p^{2}-1 \equiv 0$ $(\bmod 16)$ or $G$ is a soluble group such that $G / \operatorname{Soc}(G)$ is supersoluble.

Note that the class of all groups with every subgroup supersoluble or subnormal is a proper subclass of the one studied in Theorem A. This class was studied in [5]. The reader is also referred to [1], [2], [3], [6], [8], [10], [15], [18], and [19] for other interesting related results. 


\section{Some preliminary results}

The aim of this short section is to collect some definitions and results which will be used in the proofs of our main theorems. These results will be applied in the sequel without any further comment.

Recall that if $\mathfrak{X}$ is a class of groups, a subgroup $U$ of a group $G$ is said to be $\mathfrak{X}$-maximal in $G$ provided that

1) $U \in \mathfrak{X}$

2) if $U \leq V \leq G$ and $V \in \mathfrak{X}$, then $U=V$.

A subgroup $U$ of a group $G$ is called an $\mathfrak{X}$-projector of $G$ if $U K / K$ is $\mathfrak{X}$-maximal in $G / K$ for all normal subgroups $K$ of $G$.

A class of groups $\mathfrak{F}$ is called a formation if it is closed under taking epimorphic images and subdirect products; $\mathfrak{F}$ is said to be saturated if a group $G$ belongs to $\mathfrak{F}$ provided that the Frattini factor group $G / \Phi(G)$ belongs to $\mathfrak{F}$. According to [9] (see III, 3.10 and II, 4.1), if $\mathfrak{F}$ is a saturated formation, every group has $\mathfrak{F}$-projectors. If $\mathfrak{F}$ is a formation, each group has a smallest normal subgroup with quotient in $\mathfrak{F}$. This subgroup is called the $\mathfrak{F}$-residual of $G$ and is denoted by $G^{\mathfrak{F}}$. A classical result (see 4.2.1 in [7]) shows that if $\mathfrak{F}$ is a saturated formation, the $\mathfrak{F}$ projectors of a group with soluble $\mathfrak{F}$-residual form a conjugacy class of subgroups. A useful splitting theorem which generalises a theorem due to Higman shows that if the $\mathfrak{F}$-residual of a group $G$ is abelian, where $\mathfrak{F}$ is a saturated formation, then it is complemented by every $\mathfrak{F}$-projector of $G$ (see IV, 5.18 in [9]). As a consequence, if $U$ is an $\mathfrak{F}$-projector of $G$, then $U \cap G^{\mathfrak{F}}$ is contained in the derived subgroup of $G^{\mathfrak{F}}$.

A chief factor $H / K$ of a group $G$ is called $\mathfrak{F}$-central in $G$ if $G / C_{G}(H / K)$ belongs to $\mathfrak{F}$. The product of all normal subgroups $N$ of a group $G$ with the property that every $G$-chief factor below $N$ is $\mathfrak{F}$-central in $G, \mathfrak{F}$ a saturated formation, is called the $\mathfrak{F}$-hypercentre of $G$ and is denoted by $\mathrm{Z}_{\mathfrak{F}}(G)$. Every $G$-chief factor below $\mathrm{Z}_{\mathfrak{F}}(G)$ is $\mathfrak{F}$-central in $G, \mathrm{Z}_{\mathfrak{F}}(G)$ is contained in every $\mathfrak{F}$-projector of $E$ of $G$, and in fact $\mathrm{Z}_{\mathfrak{F}}(G)=\mathrm{C}_{E}\left(G^{\mathfrak{F}}\right)$ (see IV, 6.14 in $\left.[9]\right)$.

According to IV, 3.4 (f) in [9], the class $\mathfrak{U}$ of all supersoluble groups is a saturated formation which is locally defined by the formation function $u$ such that $u(p)$ is the formation of all abelian groups of exponent dividing $p-1$ for all primes $p$. Hence every chief factor of a supersoluble group $G$ is cyclic and $G / \mathrm{O}_{p^{\prime}, p}(G)$ is abelian of exponent dividing $p-1$ for all primes $p$. In particular, $G^{\prime}$ is nilpotent.

\section{The proofs}

Proof of Theorem A. Let $G$ be a non-soluble group in which every non-normal maximal subgroup is supersoluble. Let $S$ denote the soluble residual of $G$, that is, the smallest normal subgroup of $G$ with soluble quotient. Then $S \neq 1$, and $G$ satisfies the following properties:

1. G has exactly one non-abelian chief factor in every chief series.

Let $T$ be a normal subgroup of $G$ such that $S / T$ is a chief factor of $G$. Note that $S / T$ is non-abelian. Since $G / T$ is not nilpotent, it contains a non-normal maximal 
subgroup $H / T$. We then have that $H$ is supersoluble and so $T$ is supersoluble. Therefore $S / T$ is the unique non-abelian chief factor in every chief series of $G$ passing through $T$ and $S$. By the strengthened form of the Jordan-Hölder theorem (see A, 9.13 in [9]), $G$ has exactly one non-abelian chief factor in every chief series.

\section{2. $G / S$ is nilpotent.}

Clearly we may assume that $S$ is a proper subgroup of $G$. Let $U$ be a maximal subgroup containing $S$. Since $S$ is not soluble, it follows that $U$ is not supersoluble and so $U$ is normal in $G$. Therefore all maximal subgroups of $G / S$ are normal. This implies that $G / S$ is nilpotent.

3. Let $p$ be a prime, let $N \leq R$ be normal subgroups of $G$, and let $P / N$ be a Sylow p-subgroup of $R / N$. Assume that $P$ is not normal in $G$. Let $M$ be a maximal subgroup of $G$ containing $\mathrm{N}_{G}(P)$. Then $M$ is not normal in $G$ and, in particular, $\mathrm{N}_{G}(P)$ is supersoluble.

Assume that $M$ is normal in $G$. Then $R \cap M$ is also a normal subgroup of $G$ and $P$ is a Sylow $p$-subgroup of $R \cap M$. By the Frattini argument, $G=$ $(M \cap R) \mathrm{N}_{G}(P) \leq M$, which contradicts the maximality of $M$. Therefore $M$ is not normal in $G$.

4. Let $S / T$ be the unique non-abelian chief factor in a chief series of $G$ containing $S$. Then $S / T$ is a simple group.

Clearly we may assume that $T=1$. Then $S=S_{1} \times \cdots \times S_{n}$, where $S_{1}$ is a nonabelian simple group, $S_{i} \cong S_{1}$ for $1 \leq i \leq n$. Suppose that $S$ is not simple. Hence $n>1$. Let $p \geq 5$ be a prime dividing $\left|S_{1}\right|$ and let $P_{i}$ denote a Sylow $p$-subgroup of $S_{i}, 1 \leq i \leq n$. It clearly follows that $P=P_{1} \times \cdots \times P_{n}$ is a Sylow $p$-subgroup of $S$. Applying $\mathrm{X}, 8.13$ in [13], $\mathrm{N}_{S_{i}}\left(P_{i}\right) \neq P_{i} \mathrm{C}_{S_{i}}\left(P_{i}\right)$. Hence $\mathrm{N}_{S_{i}}\left(P_{i}\right)$ contains a non-central $p$-chief factor. Since $P$ is not normal in $G, \mathrm{~N}_{G}(P)$ is a proper subgroup of $G$ and so there exists a maximal subgroup $M$ of $G$ containing $\mathrm{N}_{G}(P)$. By Step 3, $M$ is not normal in $G$. This implies that $\mathrm{N}_{G}(P)$ is supersoluble.

On the other hand, by the Frattini argument $G=\mathrm{N}_{G}(P) S$. It follows easily that $\mathrm{N}_{G}(P)$ permutes the subgroups $S_{1}, \ldots, S_{n}$ transitively. In particular, $\mathrm{N}_{G}(P)$ is not contained in $\mathrm{N}_{G}\left(S_{1}\right)$, and hence we may choose an element $x \in \mathrm{N}_{G}(P) \backslash$ $\mathrm{N}_{G}\left(S_{1}\right)$. Let $y$ be a $p^{\prime}$-element of $\mathrm{N}_{S_{1}}\left(P_{1}\right) \backslash\left(P_{1} \mathrm{C}_{S_{1}}\left(P_{1}\right)\right)$ and consider $A=\langle x, y\rangle$. Since $S_{1}^{x}=S_{j}$ for some $j \neq 1$, we have that $y^{-1} y^{x}$ is a nontrivial $p^{\prime}$-element of $S$. We also have that $P$ is normal in $P A$. Since $[y, x]$ has $p^{\prime}$-order, if $P A$ were supersoluble we would have that $[y, x]$ centralises $P$ by A, 12.4 in [9]. Since $y^{x} \in S_{j}, y^{x}$ centralises $P_{1}$ and hence $y$ centralises $P_{1}$, contradicting the choice of $y$. It follows that $P A$ and hence $\left(\mathrm{N}_{S_{1}}\left(P_{1}\right) \times \cdots \times \mathrm{N}_{S_{n}}\left(P_{n}\right)\right)\langle x\rangle$ is not supersoluble. This contradicts the fact that $\mathrm{N}_{G}(P)$ is supersoluble. Therefore $S$ must be simple and the desired conclusion holds.

Let $N$ be the soluble radical of $G$, that is, the largest normal soluble subgroup of $G$. Since $T$ is soluble, $N \cap S=T$. It implies that $S N / N$ is the unique minimal normal subgroup of $G / N$. Since $S N / N$ is simple, $G / N$ is an almost simple group. 
5. $N$ is nilpotent. In particular, $N=\mathrm{F}(G)$, the Fitting subgroup of $G$.

Suppose that $N$ is not nilpotent. Then, for some prime $p$, there is a Sylow $p$-subgroup $P$ of $N$ which is not normal in $N$. By the Frattini argument, we have that $G=N \mathrm{~N}_{G}(P)$ and then $\mathrm{N}_{G}(P) /\left(N \cap \mathrm{N}_{G}(P)\right) \cong G / N$ is insoluble. If $M$ is a maximal subgroup of $G$ containing $\mathrm{N}_{G}(P)$, then $M$ must be normal in $G$. This contradicts Step 3.

6. $G / N \cong \mathrm{PGL}_{2}(p)$, where $p$ is a prime with $p^{2}-1 \equiv 0(\bmod 16)$.

By Theorem 4, we have that the composition factors of $G / N$ are cyclic of prime order or $\operatorname{PSL}_{2}(p)$, with $p^{2}-1 \equiv 0(\bmod 16)$. Therefore $\operatorname{Soc}(G / N)=S N / N$ is isomorphic to $\operatorname{PSL}_{2}(p)$. This means that $G / N$ is isomorphic to either $\operatorname{PSL}_{2}(p)$ or $\operatorname{Aut}\left(\mathrm{PSL}_{2}(p)\right) \cong \mathrm{PGL}_{2}(p)$. If $G / N$ were isomorphic to $\mathrm{PSL}_{2}(p)$, then we would have that all maximal subgroups of $\operatorname{PSL}_{2}(p)$ would be supersoluble, but $\operatorname{PSL}_{2}(p)$ has a subgroup isomorphic to the alternating group $A_{4}$ of degree 4 by II, 8.27 in [12]. Therefore $G / N \cong \operatorname{PGL}_{2}(p)$ with $p^{2}-1 \equiv 0(\bmod 16)$.

\section{Every chief factor of $G$ below $T$ is cyclic of order 2 .}

Let $A / B$ be a chief factor of $G$ below $T$. We assume without loss of generality that $B=1$. Then $A$ is a minimal normal subgroup of $G$ contained in $S$. Since $N$ is the Fitting subgroup of $G, N \leq \mathrm{C}_{G}(A)$. Therefore $\mathrm{C}_{G}(A) / N$ is a normal subgroup of $G / N$.

Assume that $N=\mathrm{C}_{G}(A)$. Let $q$ be the prime dividing $|A|$. Suppose that $q \neq 2$. Let $G_{2}$ be a Sylow 2-subgroup of $G$ and set $C:=N G_{2}$. It is clear that $C / N$ is a Sylow 2-subgroup of $G / N$ and $C / N$ is non-abelian because the Sylow 2-subgroups of $\mathrm{PGL}_{2}(q)$ contain Sylow 2-subgroups of $\mathrm{PSL}_{2}(q)$ and, by II, 8.27 in [12], the latter are dihedral groups. Moreover $C / N$ is not a subgroup of $S N / N$. Therefore $C$ is contained in a non-normal maximal subgroup of $G$ and so $C$ is supersoluble. Consequently $A$, regarded as a $G_{2}$-module over $\mathrm{GF}(q)$, is a direct sum of one-dimensional submodules. This implies that $C / N$ is abelian. This contradiction yields $q=2$.

Let $P / N$ be a Sylow $p$-subgroup of $G / N$. Let $K / N=\mathrm{N}_{G / N}(P / N)$. It is clear that $K / N \not \leq S N / N$, since otherwise $P / N$ is a Sylow $p$-subgroup of $S N / N$ and, by the Frattini argument, $G / N=(S N / N)(K / N) \leq S N / N$. Moreover $K \neq G=S K$. Hence $K$ must be supersoluble and, since $A$ is a 2-group, every chief factor of $K$ below $A$ is central in $K$. Let $P_{0}$ be a Sylow $p$-subgroup of $P$. By A, 12.3 in [9], $P_{0} \leq \mathrm{C}_{G}(A)=N$. This is contrary to our assumption. Therefore $N$ is a proper subgroup of $\mathrm{C}_{G}(A), S$ is contained in $\mathrm{C}_{G}(A)$ and $\left|G: \mathrm{C}_{G}(A)\right| \leq 2$. If $|A|$ is a power of 2 , then $G=\mathrm{C}_{G}(A)$ as $\mathrm{O}_{2}\left(G / \mathrm{C}_{G}(A)\right)=1$ (see A, 13.6 in [9]). If $|A|$ is a power of a prime $q \neq 2$, then $A$ is cyclic because $2 \mid q-1$ (see B, 9.8 in [9]). Assume that $A$ is not contained in the Frattini subgroup $\Phi(S)$ of $S$. Then $S=A R$ for some maximal subgroup $R$ of $S$. Since $A$ is central in $S$, we have that $R$ is normal in $S$. This contradicts the fact that $S$ is perfect. Consequently, every chief factor $C / D$ of $G$ below $T$ is cyclic, $S$ centralises $C / D$ and $C / D \leq \Phi(S / D)$.

Suppose that $T$ is not a 2-group and let $D$ be a normal subgroup of $G$ such that $T / D$ is a chief factor of $G$ of odd order. Then $T / D$ is cyclic and $T / D \leq$ 
$\mathrm{Z}(S / D) \cap \Phi(S / D)$. This implies that the Schur multiplier of $S / T$ is divisible by an odd prime. This is a contradiction (see, for instance, V, 25.7 in [12]). Therefore $T$ is a 2-group.

8. $S$ is isomorphic to $\mathrm{PSL}_{2}(p)$ or $\mathrm{SL}_{2}(p)$.

Assume that $S$ is not isomorphic to $\operatorname{PSL}_{2}(p)$. Let $D$ be a normal subgroup of $G$ such that $T / D$ is a chief factor of $G$. By Step 7, $T / D$ is of order 2 , and $S / D$ is a Frattini central extension of $S / T \cong \mathrm{PSL}_{2}(p)$. By V, 23.5, 23.6 and 25.7 in [12], since $\mathrm{SL}_{2}(p)$ is a Frattini central extension of $\operatorname{PSL}_{2}(p)$, it follows that $S / D \cong \mathrm{SL}_{2}(p)$. Assume now that $D / E$ is a chief factor of $G$. Again $D / E$ is central in $S / E$ and $D / E$ is contained in $\mathrm{Z}(S / E) \cap \Phi(S / E)$. This contradicts the fact that the Schur multiplier of $S / D \cong \mathrm{SL}_{2}(p)$ is trivial (see $\mathrm{V}, 25.5$ in [12]). Therefore $S$ is isomorphic to $\mathrm{PSL}_{2}(p)$ or $\mathrm{SL}_{2}(p)$.

Consequently, if $G$ is an insoluble group whose non-normal maximal subgroups are supersoluble, then $G$ satisfies (a) and (b) of the Statement 1 of the theorem.

9. The soluble case.

Assume now that $G$ is a non-supersoluble soluble group whose non-normal maximal subgroups are supersoluble. Let $1 \neq A$ denote the supersoluble residual of $G$, that is, the smallest normal subgroup of $G$ with supersoluble quotient. Then:

Step 9.1. A is a p-group for a prime $p, A / A^{\prime}$ is a complemented non-cyclic chief factor of $G$, and all chief factors of $G$ containing $A$ or contained in $A^{\prime}$ are cyclic. Moreover, A has nilpotency class at most two.

By IV, 5.8 in [9], there exists a maximal subgroup $M$ of $G$ such that $G=$ $\mathrm{F}(G) M$ and $G / R$ is not supersoluble, where $R$ is the core of $M$ in $G$. Since $M$ cannot be normal in $G$, it follows that $M$ is supersoluble. Consequently $M$ is a supersoluble projector of $G$. In particular, $M /(\mathrm{F}(G) \cap M)$ is supersoluble and so is $M \mathrm{~F}(G) / \mathrm{F}(G)=G / \mathrm{F}(G)$. Then $G=A M$ and $A$ is contained in $\mathrm{F}(G)$. Moreover there exists a minimal normal subgroup $I / \Phi(G)$ of $G / \Phi(G)$ such that $G / \Phi(G)=(I / \Phi(G))(M / \Phi(G))$ because $\mathrm{F}(G) / \Phi(G)$ is a direct product of minimal normal subgroups of $G / \Phi(G)$ by A, 10.6 in [9]. Let $p$ be the prime dividing the order of $I / \Phi(G)$. Since $A \Phi(G)$ is contained in $I$ but $A$ is not contained in $\Phi(G)$, it follows that $I=A \Phi(G)$. On the other hand, by IV, 5.18 in [9], we have that $A \cap M$ is contained in $A^{\prime}$. This implies that $A^{\prime}=A \cap M=\Phi(A)=A \cap \Phi(G)$ because $A$ is nilpotent. Consequently, $A / A^{\prime}$ is a $p$-chief factor of $G$ which is $G$-isomorphic to $I / \Phi(G)$. In particular, $A / A^{\prime}$ is a non-cyclic $p$-group. Moreover, since $A$ is nilpotent, $A^{\prime} \leq \Phi(A)$ and so $A$ itself is a $p$-group. Since $A^{\prime}$ is a normal subgroup of $G$ and $A^{\prime}=A \cap M, A^{\prime} \leq \operatorname{Core}_{G}(M)=\mathrm{Z}_{\mathfrak{U}}(G)$, the supersoluble hypercentre of $G$, by $\mathrm{V}, 2.4$ and 4.2 in [9], which is contained in $\mathrm{C}_{G}(A)$. It follows that $A^{\prime} \leq \mathrm{Z}(A)$ and so $A$ has nilpotency class at most two. Moreover, since $A^{\prime} \leq \mathrm{Z}_{\mathfrak{U}}(G)$, all chief factors of $G$ below $A^{\prime}$ are cyclic.

Let $Z=\mathrm{Z}_{\mathfrak{U}}(G)$ be the supersoluble hypercentre of $G$. Then:

Step 9.2. $Z$ contains no non-central complemented chief factors of $G$. 
Let $H / K$ be a complemented chief factor of $G$ below $Z$. Then there exists a subgroup $W$ of $G$ containing $K$ such that $G / K=(H / K)(W / K)$ and $H \cap W=K$. Since $G / K$ is a non-supersoluble quotient of $G / H$, it follows that $G / H$ is not supersoluble. This implies that every complement of $H / K$ in $G$ is a non-supersoluble maximal subgroup of $G$. Our hypothesis implies that every complement of $H / K$ is normal in $G$ and hence $H / K$ is central in $G$.

We suppose in the sequel that $G / \mathrm{C}_{G}\left(A / A^{\prime}\right)$ is not nilpotent. Since we have that $G / \mathrm{C}_{G}\left(A / A^{\prime}\right)$ is isomorphic to $\left(G / A^{\prime}\right) / \mathrm{C}_{G / A^{\prime}}\left(A / A^{\prime}\right)$, there is no loss of generality in assuming that $A^{\prime}=1$ and, hence, that $A$ is abelian. Thus $G=M A=M \mathrm{C}_{G}(A)$ and $\mathrm{C}_{G}(A)=A M \cap \mathrm{C}_{G}(A)=A \mathrm{C}_{M}(A)$. It follows that $G / \mathrm{C}_{G}(A)=M A / \mathrm{C}_{G}(A) \cong$ $M / \mathrm{C}_{M}(A)$. Furthermore, $\mathrm{C}_{M}(A)$, which coincides with the supersoluble hypercentre $Z$ of $G$, is centralised by $A$ and so $\mathrm{C}_{M}(A)$ is a normal subgroup of $G$. There is no loss of generality if we assume that $\mathrm{C}_{M}(A)=1$ (otherwise, we can take quotients by the normal subgroup $\left.\mathrm{C}_{M}(A)\right)$ and $A$ is a faithful and irreducible $M$-module over $\mathrm{GF}(p)$.

Let $B=M^{\mathfrak{N}}$ be the nilpotent residual of $M$ and let $C$ be a nilpotent projector (a Carter subgroup) of $M$. Then:

Step 9.3. $B$ is a $p^{\prime}$-group, and if $K$ is a normal subgroup of $M$ properly contained in $B$ such that $B / K$ is a chief factor of $M$, then $K=\mathrm{C}_{B}(C)$.

We have that $M=B C$. Since $M$ is supersoluble, $M^{\prime}$ is nilpotent and so $B$, which is contained in $M^{\prime}$, is also nilpotent. By $\mathrm{B}, 3.12$ in $[9], \mathrm{O}_{p}(B) \leq \mathrm{O}_{p}(M)=1$. Consequently $B$ is a $p^{\prime}$-group.

Suppose that $K$ is a normal subgroup of $M$ properly contained in $B$ such that $B / K$ is a chief factor of $M$. Consider the subgroup $Y=A(K C)$ of $G$. We have that $Y \cap M=K C$ and the normal closure $\left\langle C^{M}\right\rangle$ of $C$ in $M$ coincides with $M$ by I, 6.21, III, 3.22, and III, 4.9 in [9]. Note that $Y$ is a proper subgroup of $G$, because otherwise $M=Y \cap M=K C$, which would imply that $M / K=K C / K$ is nilpotent and so $B \leq K$, contrary to the choice of $K$. Hence $Y$ is contained in a maximal subgroup $M_{0}$ of $G$. Suppose that $M_{0}$ is a normal subgroup of $G$. Then $C \leq M_{0} \cap M$, which is a normal subgroup of $M$. This contradicts that $\left\langle C^{M}\right\rangle=M$. Hence $M_{0}$ is not a normal subgroup of $G$. Therefore $M_{0}$ is supersoluble and so $Y$ is supersoluble. Since $M$ acts faithfully on $A, \mathrm{O}_{p^{\prime}}(Y)=1$. Hence $Y / \mathrm{O}_{p^{\prime}, p}(Y)=$ $Y / \mathrm{O}_{p}(Y)$ is an abelian group of exponent dividing $p-1$. In particular, $Y^{\prime}$ is a $p$-group and so $(K C)^{\prime}$ is a $p$-group. However $(K C)^{\prime} \leq M^{\prime}$ and $M^{\prime}$ is a nilpotent group such that $\mathrm{O}_{p}\left(M^{\prime}\right) \leq \mathrm{O}_{p}(M)=1$. Hence $(K C)^{\prime}=1$ and $K C$ is abelian. Hence $K \leq \mathrm{C}_{B}(C) \leq B$. Since $B / K$ has prime order and $B$ cannot centralise $C$, it follows that $K=\mathrm{C}_{B}(C)$.

Step 9.4. $M$ is isomorphic to a nontrivial semidirect product $[B](T \times P)$, where $T$ is an abelian group of exponent dividing $p-1, P$ is a p-group, and $B$ is a cyclic group of order $q$ for a prime $q \neq p$.

Let $L / T$ be a chief factor of $M$ such that $T<L \leq K$. Since $M$ is supersoluble, $B$ is contained in the Fitting subgroup $\mathrm{F}(M)$ of $M$, which centralises $L / T$. Since $C$ centralises $L / T$, it turns out that $L / T$ is central in $M$. Therefore every chief factor of $M$ below $K$ is central in $M$. It follows that $K \leq \mathrm{Z}_{\infty}(M)$, which is contained 
in $C$. Hence $C$ is a maximal subgroup of $M$. On the other hand, $C \cap B$ is contained in $B^{\prime}$ and $B^{\prime} \leq \Phi(B)$ because $B$ is nilpotent. Therefore $K=\Phi(B)$ and, since $B / K$ is cyclic, $B$ is cyclic. Therefore $B \cap C=K=1$. Thus $|B|=q \neq p$. Moreover $M=[B] C$, where $C$ is nilpotent. We can factorise $C=C_{p} \times C_{p^{\prime}}$, where $C_{p}$ is the Sylow $p$-subgroup of $C$ and $C_{p^{\prime}}$ is the Hall $p^{\prime}$-subgroup of $C$. Since $C$ is a maximal subgroup of $M$ and $C$ is not normal in $M, A C$ is a nonnormal maximal subgroup of $G$. Thus $A C$ is a supersoluble group. Moreover $\mathrm{O}_{p^{\prime}}(A C)=1$ because $M$ acts faithfully on $A$. Hence $A C / \mathrm{O}_{p}(A C)$ is an abelian group of exponent dividing $p-1$. Consequently $C_{p^{\prime}}$ is an abelian group of exponent dividing $p-1$. Therefore $M=[B]\left(C_{p^{\prime}} \times C_{p}\right)$, as desired.

Step 9.5. The nilpotent residual of $G / A$ is a q-group.

We can assume, without loss of generality, that $A^{\prime}=1$. Hence $G=A M$ with $A \cap M=1$ and so $M \cong G / A$. We know that the nilpotent residual of $M / \mathrm{C}_{M}(A)=$ $M / Z$, which coincides with $M^{\mathfrak{N}} Z / Z$, is a cyclic group of order $q$. It follows that $M^{\mathfrak{N}} /\left(M^{\mathfrak{N}} \cap Z\right)$ is a cyclic group of order $q$. Moreover, $M^{\mathfrak{N}}$ is nilpotent because $M$ is supersoluble. Assume that $M^{\mathfrak{N}}$ is not a $q$-group. Then $M^{\mathfrak{N}}=\mathrm{O}_{q}\left(M^{\mathfrak{N}}\right) \times \mathrm{O}_{q^{\prime}}\left(M^{\mathfrak{N}}\right)$ and $\mathrm{O}_{q^{\prime}}\left(M^{\mathfrak{N}}\right) \leq M^{\mathfrak{N}} \cap Z$. Let $T$ be a normal subgroup of $M$ such that $T \leq$ $\mathrm{O}_{q^{\prime}}\left(M^{\mathfrak{N}}\right)$ and $\mathrm{O}_{q^{\prime}}\left(M^{\mathfrak{N}}\right) / T$ is a chief factor of $M$. Then $M^{\mathfrak{N}} / T \mathrm{O}_{q}\left(M^{\mathfrak{N}}\right)$ is a chief factor of $M$. Moreover, it is $M$-isomorphic to the chief factor $\mathrm{O}_{q^{\prime}}\left(M^{\mathfrak{N}}\right) / T$. By the strengthened form of the Jordan-Hölder theorem (see A, 9.13 in [9]), since the unique chief factor of order different from $q$ between $T$ and $M^{\mathfrak{N}}$ is $M^{\mathfrak{N}} / T \mathrm{O}_{q}\left(M^{\mathfrak{N}}\right)$, which is $M$-isomorphic to $\mathrm{O}_{q^{\prime}}\left(M^{\mathfrak{N}}\right) / T$, we have that either both factors are Frattini or both factors are complemented in $G$. Obviously, $M^{\mathfrak{N}} / T \mathrm{O}_{q}\left(M^{\mathfrak{N}}\right)$ cannot be Frattini, because then $M^{\mathfrak{N}} \leq T \mathrm{O}_{q}\left(M^{\mathfrak{N}}\right)$. Therefore it is complemented, and so is $M^{\mathfrak{N}} / T \mathrm{O}_{q}\left(M^{\mathfrak{N}}\right)$. But since $M^{\mathfrak{N}} \leq Z$, this chief factor must be central. It follows that $M^{\mathfrak{N}} / T \mathrm{O}_{q}\left(M^{\mathfrak{N}}\right)$ is central, which implies again that $M^{\mathfrak{N}} \leq T \mathrm{O}_{q}\left(M^{\mathfrak{N}}\right)$. Hence $M^{\mathfrak{N}}$ must be a $q$-group, as desired.

This completes the proof of the soluble case.

\section{The converse.}

Assume now that $G$ is an insoluble group such that $G / \mathrm{F}(G) \cong \mathrm{PGL}_{2}(p)$ with $p$ a prime such that $p^{2}-1 \equiv 0(\bmod 16)$, the soluble residual $S$ is isomorphic to $\operatorname{PSL}_{2}(p)$ or $\operatorname{SL}_{2}(p)$, and $G / S$ is nilpotent. Let $U$ be a non-normal maximal subgroup of $G$. Then $G=S U$. Assume further that $U$ contains a minimal normal subgroup $A$ of $G$. If $A$ were non-abelian, then $A \cong \operatorname{PSL}_{2}(p)$ and so $A$ would be contained in $S$. This would imply that $A=S$. This contradiction yields that $A$ is abelian. Hence $A$ is central in $G$ and $G / A$ is an insoluble group satisfying the hypotheses of the theorem. It follows then that $U / A$ is supersoluble by induction. Since $A$ is central in $G$, we have that $U$ is supersoluble.

Therefore, we can assume that $\operatorname{Core}_{G}(U)=1$. Then $G$ is a primitive group. Since $\Phi(G)$ is trivial, it follows that $S$ is isomorphic to $\operatorname{PSL}_{2}(p)$. It implies that $S=\operatorname{Soc}(G)$ and $\mathrm{C}_{G}(S)=1=\mathrm{F}(G)$ (see for instance 1.1.7 in [7]). Therefore $G \cong$ $\mathrm{PGL}_{2}(p)$, and we must only check that $\mathrm{PGL}_{2}(p)$ has all its non-normal maximal subgroups supersoluble. 
Consequently, we shall assume in the sequel that $G=\mathrm{PGL}_{2}(p)$, with $p$ a prime such that $p^{2}-1 \equiv 0(\bmod 16)$. We denote $S=\operatorname{PSL}_{2}(p)$.

Let $K$ be a proper subgroup of $G$ such that $G=S K$. Then $K \cap S$ is a proper subgroup of $S$ such that $|K: K \cap S|=2$. The proper subgroups of $S$ are known; see II, 8.27 in [12]. We have that $K \cap S$ is isomorphic to one of the following groups: an elementary abelian $p$-group, a cyclic group of order $z$ dividing $(p \pm 1) / 2$, a dihedral group of order $2 z$ with $z$ dividing $(p \pm 1) / 2$, the semidirect product of a subgroup of order $p$ with a cyclic subgroup of order $t$ where $t$ divides $p-1, A_{4}, \Sigma_{4}$, or $A_{5}$. If $K \cap S$ is an elementary abelian $p$-group or a cyclic group of order $z$ dividing $(p \pm 1) / 2$ or a dihedral group of order $2 z$ with $z$ dividing $(p \pm 1) / 2$, then $K$ is supersoluble (recall that the subgroups of the cyclic group of order $z$ are characteristic in the dihedral group of order $2 z$ ). Now assume that $K \cap S$ is the semidirect product of a subgroup of order $p$ with a cyclic subgroup of order $t$, where $t$ divides $p-1$. If $t \neq 2$, then we can choose an element $x$ of order 2 of $K \backslash S$ such that $\langle x\rangle$ permutes with $C_{t}$. We have that $x$ acts on $C_{p}$ and so the subgroup generated by $x$ and $C_{t}$ acts as an abelian group on $C_{p}$. It follows that $K$ is supersoluble. Assume now that $t=2$. Then clearly $K$ has a normal series with cyclic factors because $K \cap S$ is dihedral; in particular, $K$ is supersoluble. Suppose that $K \cap S$ is isomorphic to $A_{4}$ or $\Sigma_{4}$. Let $V$ be the unique minimal normal subgroup of $K \cap S$, which is elementary abelian of order 4 and normalised by $K$. It follows from II, 8.27 in [12] that $\mathrm{N}_{G}(V) \leq S$. This contradicts the fact that $K$ supplements $S$ in $G$. Assume that $K \cap S \cong A_{5}\left(\right.$ in this case, $\left.p^{2}-1 \equiv 0(\bmod 5)\right)$. Consider an element $x$ of order a power of 2 such that $K=(K \cap S)\langle x\rangle$. If $x$ centralises $K \cap S$, then we can find an elementary abelian subgroup $V$ of order 4 contained in $K \cap S$ and then $x \in \mathrm{N}_{G}(V) \leq S$, a contradiction. Therefore $x$ does not centralise $K \cap S$. Hence $x$ induces a nontrivial automorphism of $A_{5}$. The group $A_{5}$ has 5 conjugacy classes of elementary abelian subgroups of order 4 . Since $x$ has order a power of 2 , one of these subgroups $V$ must be normalised by $x$. But then $x \in \mathrm{N}_{G}(V) \leq S$. This contradiction proves that all proper supplements of $S$ in $G$ must be supersoluble.

Since every non-normal maximal subgroup of $G$ is a proper supplement of $S$ in $G$, we conclude that every non-normal maximal subgroup of $G$ is supersoluble.

Suppose now that $G$ is a soluble group satisfying conditions (a) and (b) of Statement 2. We shall prove that every non-normal maximal subgroup of $G$ is supersoluble. We argue by induction on the order of $G$. Since the hypotheses of $G$, as enunciated in the statement of the theorem, are inherited by $G / A^{\prime}$ and $A^{\prime}$ is contained in $Z$, we may assume that $A^{\prime}=1$. Then $A$ is a minimal normal p-subgroup of $G, p$ a prime, which is complemented in $G$ by every supersoluble projector of $G$, and $C_{G}(A)=A Z$. Let $U$ be a non-normal maximal subgroup of $G$. Suppose that $G=A U$. Then $U$ is a complement of $A$ in $G$ and so $U$ is a supersoluble projector of $G$. Hence we may suppose that $U$ contains $A$. Assume that $Z$ is not contained in $U$. Then $G=U Z$ and there exists a chief factor $H / K$ of $G$ such that $K$ is contained in $U$ and $G=U H$. Hence $H / K$ is a complemented chief factor of $G$. The hypotheses on $G$ imply that $H / K$ is central in $G$ and so $U$ is normal in $G$. Therefore we may assume that $Z$ is contained in $U$. This implies 
that $C=\mathrm{C}_{G}(A)$ is a subgroup of $U$. This means that $G / C$ is not nilpotent and so $G / C$ satisfies condition (b) of Statement 2. Let $V$ be a subgroup of $U$ contained in a supersoluble projector of $G$ such that $U=A V$. We prove that every $U$-chief factor below $A$ is cyclic. There is no loss of generality in assuming that $C \cap V=1$ since $C \cap V$ is a normal subgroup of $G$ which is contained in the supersoluble hypercentre of $U$. Then $\mathrm{C}_{G}(A)=A$ and the nilpotent residual of $G / A$ is a cyclic group of order $q$ for some prime $q \neq p$. Suppose that $q$ divides the order of $V$. Then $U$ contains the nilpotent residual of $G$ and so $U$ is normal in $G$. Hence we may assume that $V$ is a $q^{\prime}$-group. But then the hypothesis on $G$ implies that $V=W \times Y$ is a direct product of an abelian group $W$ of exponent dividing $p-1$ and a $p$-group $Y$. Applying A, 13.18 (b) in [9] and B, 9.8 in [9], we see that every $U$-chief factor below $A$ is cyclic. Consequently, $U$ is supersoluble.

Proof of Theorem B. Let $G$ be a primitive group. Assume that every core-free maximal subgroup of $G$ is supersoluble. Then it is clear that $\operatorname{Soc}(G)$ cannot be the product of two different minimal normal subgroups of $G$, because in this case each one is complemented by every core-free maximal subgroup of $G$ (see 1.1.7 in [7]). Hence, either $\operatorname{Soc}(G)$ is a non-abelian minimal normal subgroup of $G$ or $\operatorname{Soc}(G)$ is abelian and complemented by every core-free maximal subgroup of $G$. In the latter case, $G$ is a non-supersoluble group with $G / \operatorname{Soc}(G)$ supersoluble. Suppose now that $S=\operatorname{Soc}(G)$ is a non-abelian minimal normal subgroup of $G$. In this case, $S$ is the soluble residual of $G$. The argument used in Theorem A to prove Step 4 can also be applied in this case. We conclude then that $S$ is a non-abelian simple group. Note that $S$ is a proper subgroup of $G$ by Theorem 3. Since $S$ is supplemented by every core-free maximal subgroup of $G$, it follows that $G / S$ is supersoluble. This implies that for every maximal subgroup $M$ of $G, \operatorname{Sec}(M)$ is supersoluble (see [14]). By Theorem 4, we have that the composition factors of $G$ are cyclic of prime order or $\mathrm{PSL}_{2}(p)$ with $p^{2}-1 \equiv 0(\bmod 16)$. This implies that $S$ is isomorphic to $\mathrm{PSL}_{2}(p)$. Since $\mathrm{C}_{G}(S)=1$, we have that $G$ is isomorphic to $\operatorname{Aut}(S) \cong \mathrm{PGL}_{2}(p)$ with $p^{2}-1 \equiv 0(\bmod 16)$.

Conversely, assume that $G$ is isomorphic to $\operatorname{PGL}_{2}(p)$ with $p^{2}-1 \equiv 0(\bmod 16)$. Then every core-free maximal subgroup of $G$ is a proper supplement of $\operatorname{Soc}(G) \cong$ $\operatorname{PSL}_{2}(p)$. By what we have proved already in Step 10 of Theorem A, every corefree maximal subgroup of $G$ is supersoluble. If $G$ is soluble and $G / \operatorname{Soc}(G)$ is supersoluble, then the result also follows as every core-free maximal subgroup of $G$ is isomorphic to $G / \operatorname{Soc}(G)$. This completes the proof of Theorem B.

\section{Examples}

The purpose of this section is to give examples showing that, in some sense, the characterisation of Theorem $\mathrm{A}$ is best possible.

Example 1. Condition (b) of Statement 2 in Theorem A cannot be improved to "if $Q$ is the nilpotent residual of $G / A$ and $C$ is a complement of $Q / Q^{\prime}$ in $G / A$, then the the Hall $p^{\prime}$-subgroup of $C$ is abelian of exponent dividing $p-1$ ". Let $V_{7}$ be an irreducible and faithful module for $C_{3}$ over the field of 7 elements. Let $X=\left[V_{7}\right] C_{3}$ 
be the corresponding semidirect product and let $E$ be an extraspecial group of order 27 and exponent 9. Consider an irreducible module $V_{13}$ for $X \times E$ over the field of 13 elements such that the kernel of the action is $E$. Let $G=\left[V_{13}\right](X \times E)$ be the corresponding semidirect product. Then $A=V_{13}, A^{\prime}=1, M=X \times E$, $Q=V_{7}, Q^{\prime}=1$, and $C=C_{3} \times E$. Hence $C$ is not abelian of exponent dividing 12 .

Example 2. Nor can we improve the condition about the nilpotent residual of $G / A$ to " $G / A$ is a cyclic $q$-group" if $G$ is soluble and $G / \mathrm{C}_{G}\left(A / A^{\prime}\right)$ is not nilpotent. Let $S=\left\langle s_{1}, s_{2}\right\rangle$ be the extraspecial group of order 27 and exponent 3 . Let $a$ be an automorphism of order 2 of $G$ given by $s_{2}^{a}=s_{2}$ and $s_{3}^{a}=s_{3}^{2}$, so that $\left[s_{2}, s_{3}\right]^{a}=\left[s_{2}, s_{3}\right]^{2}$. Let $M=[S]\langle a\rangle$ be the corresponding semidirect product and let $V_{7}$ be an irreducible module for $M$ with kernel $\left\langle\left[s_{2}, s_{3}\right]\right\rangle$ over the field of 7 elements. Let $G=\left[V_{7}\right] M$ be the corresponding semidirect product. Then $A=V_{7}$, $A^{\prime}=1, G / A \cong M$ is supersoluble, and the nilpotent residual of $M$ is $\left\langle s_{3},\left[s_{2}, s_{3}\right]\right\rangle$, which is a non-cyclic 3-group.

Example 3. The condition " $Z$ contains no non-central complemented chief factors of $G$ " cannot be removed. The cyclic group $C_{6}$ of order 6 can be regarded as a subgroup of the automorphism groups of the cyclic groups $C_{31}$ and $C_{7}$ of orders 31 and 7 , respectively. Hence $C_{6}$ acts on $C_{31} \times C_{7}$. Let $M=\left[C_{31} \times C_{7}\right] C_{6}$ be the corresponding semidirect product. The nilpotent residual of $M$ is $C_{31} \times C_{7}$. Now let $A$ be an irreducible module for $M$ with kernel $C_{31}$ over the field of 43 elements. The corresponding semidirect product $G=[A] M$ with this action has a maximal subgroup $[A]\left(\left[C_{7}\right] C_{6}\right)$ of index 31 which is neither normal nor supersoluble, which is a complement of the supersoluble hypercentre $C_{31}$. All the other conditions of Theorem A are satisfied.

Acknowledgements. The authors are indebted to the referee, whose smart and interesting comments have substantially improved the results of the paper.

\section{References}

[1] AsaAd, M.: Finite groups some of whose $n$-maximal subgroups are normal. Acta Math. Hungar. 54 (1989) no. 1-2, 9-27.

[2] Asadd, M.: On the solvability of finite groups. Comm. Algebra 37 (2009) no. 2, 719-723.

[3] Asand, M.: A condition for the supersolvability of finite groups. Comm. Algebra 38 (2010), no. 10, 3616-3620.

[4] BAer, R.: Situation der Untergruppen und Struktur der Gruppe. S. B. Heidelberg. Akad. Wiss. 2 (1933), 12-17.

[5] Ballester-Bolinches, A. And Cossey, J.: Finite groups with subgroups supersoluble or subnormal. J. Algebra 321 (2009) no. 7, 2042-2052.

[6] Ballester-Bolinches, A., Cossey, J. and Esteban-Romero, R.: On abnormal maximal subgroups of finite groups. In Ischia Group Theory 2008, 1-6. World Sci. Publ., Hackensack, NJ, 2009.

[7] Ballester-Bolinches, A. And Ezquerro, L. M.: Classes of finite groups. Mathematics and its Applications 584, Springer, Dordrecht, 2006. 
[8] Beidleman, J. C. And Heineken, H.: A note on intersections of maximal $\mathcal{F}$-subgroups. J. Algebra 333 (2011), 120-127.

[9] Doerk, K. and Hawkes, T.: Finite soluble groups. De Gruyter Expositions in Mathematics 4, Walter de Gruyter, Berlin, 1992.

[10] Evtukhova, S. M. And Monakhov, V.S.: Finite groups with supersolvable subgroup cofactors. Vetsī Nats. Akad. Navuk Belarusī Ser. Fiz.-Mat. Navuk 2008, no. 4, $53-57$.

[11] Huppert, B.: Normalteiler und maximale Untergruppen endlicher Gruppen. Math. Z. 60 (1954), 409-434.

[12] Huppert, B.: Endliche Gruppen I. Die Grundlehren der Mathematischen Wissenschaften 134, Springer-Verlag, Berlin-New York, 1967.

[13] Huppert, B. and Blackburn, N.: Finite groups III. Grundlehren der Mathematischen Wissenschaften 243, Springer-Verlag, Berlin-New York, 1982.

[14] Li, S. ANd Shi, W.: A note on the solvability of groups. J. Algebra 304 (2006), no. $1,278-285$.

[15] Lukyanenko, V. O., Netbay, O. V. and Skiba, A. N.: Finite groups in which every non-abnormal subgroup is $X_{s}$-permutable. Vetsī Nats. Akad. Navuk Belarusī Ser. Fiz.-Mat. Navuk 2009, no. 4, 25-29.

[16] Rose, J. S.: The influence on a finite group of its proper abnormal structure. J. London Math. Soc. 40 (1965), 348-361.

[17] Schmidt, O. J.: Über Gruppen, deren sämtliche Teiler spezielle Gruppen sind. Mat. Sbornik 31 (1924), 366-372.

[18] Shemetkov, L. A. And Skiba, A. N.: Formations of algebraic systems. Nauka, Moscow, 1989.

[19] SkibA, A. N.: On the intersection of all maximal $\mathfrak{F}$-subgroups of a finite group. J. Algebra 343 (2011), 173-182.

Received February 15, 2012; revised July 3, 2012.

Adolfo Ballester-Bolinches: Departament d'Àlgebra, Universitat de València, Dr. Moliner, 50, 46100 Burjassot, València, Spain.

E-mail: Adolfo.Ballester@uv.es

John Cossey: Mathematics Department, Mathematical Sciences Institute, Australian National University, Canberra, ACT 0200, Australia.

E-mail: John.Cossey@anu.edu.au

Ramón Esteban-Romero: Institut Universitari de Matemàtica Pura i Aplicada, Universitat Politècnica de València, Camí de Vera s/n, 46022 València, Spain. Current address: Departament d'Àlgebra, Universitat de València, Dr. Moliner 50, 46100 Burjassot, València, Spain.

E-mail: resteban@mat.upv.es, ramon.esteban@uv.es

This work was supported by the grant MTM2010-19938-C03-01 from the MICINN (Spanish government). The first author was also supported by the project NSFC 11271085. 